\title{
Enhanced Strange Particle Yields - Signal of a Phase of Massless Particles ?
}

\author{
Sven Soff* ${ }_{\sharp}$, D. Zschiesche $\ddagger$, M. Bleicher $\ddagger$, C. Hartnack $\|$, \\ M. Belkacem ${ }^{+}$, L. Bravinaף, E. Zabrodinף, S.A. Bass§, \\ H. Stöcker \\ *Brookhaven National Laboratory, Physics Department, P.O. Box 5000, Upton, \\ New York 11973-5000, USA \\ \# Gesellschaft für Schwerionenforschung, Postfach 110552, D-64220 Darmstadt, \\ Germany \\ ‡Institut für Theoretische Physik der J.W.Goethe-Universität \\ Postfach 111932, D-60054 Frankfurt am Main, Germany \\ || SUBATECH, École des Mines, F-44072 Nantes, France \\ + School of Physics and Astronomy, University of Minnesota, Minneapolis, MN \\ 55455, USA \\ ๆInstitut für Theoretische Physik, Universität Tübingen \\ Auf der Morgenstelle 14, D-72076 Tübingen \\ $\S$ Department of Physics, Duke University, Durham, NC 27708, USA
}

\begin{abstract}
The yields of strange particles are calculated with the UrQMD model for $\mathrm{p}, \mathrm{Pb}(158 A \mathrm{GeV}) \mathrm{Pb}$ collisions and compared to experimental data. The yields are enhanced in central collisions if compared to proton induced or peripheral $\mathrm{Pb}+\mathrm{Pb}$ collisions. The enhancement is due to secondary interactions. Nevertheless, only a reduction of the quark masses or equivalently an increase of the string tension provides an adequate description of the large observed enhancement factors (WA97 and NA49). Furthermore, the yields of unstable strange resonances as the $\Lambda^{*}$ or the $\phi$ are considerably affected by hadronic rescattering of the decay products.
\end{abstract}

\section{Introduction}

Strange particle yields are most interesting and useful probes to examine excited

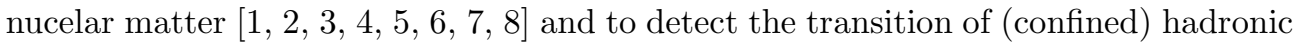
matter to quark-gluon-matter (QGP). The relative enhancement of strange and especially multistrange particles and the hadron ratios in central heavy ion collisions with respect to peripheral or proton induced interactions have been suggested as a signature for the transient existence of a QGP-phase [1]. The production mechanism of $s \bar{s}$ pairs via gluon fusion $(g g \rightarrow s \bar{s})$ [1] in an equilibrated (gluon rich) plasma phase should allow for equilibration times much shorter (a few $\mathrm{fm} / \mathrm{c}$ ) than a thermally equilibrated hadronic fireball of $T \sim 160 \mathrm{MeV}$.

Measurements by the WA97 and the NA49 collaborations clearly demonstrated the relative enhancement of the (anti-)hyperon yields $(\Lambda, \Xi, \Omega)$ in $\mathrm{Pb}-\mathrm{Pb}$ collisions as compared to $\mathrm{p}-\mathrm{Pb}$ collisions 6, 7 . The observed enhancement increases with the strangeness content $(|S|=1,2,3)$ of the probe under investigation [6, 7]. For the $\left(\Omega^{-}+\overline{\Omega^{-}}\right)$-yield the enhancement factor is as large as 15 ! 
Here, strangeness production is investigated within a microscopic transport model: hadronic and string degrees of freedom are employed in the Ultrarelativistic Quantum Molecular Dynamcis model (UrQMD) [9]. The strange baryon yields for $\mathrm{Pb}(158 A \mathrm{GeV}) \mathrm{Pb}$ collisions are computed vs. centrality and for $\mathrm{p}(158 \mathrm{GeV}) \mathrm{Pb}$ collisions. The observed total yields of $\Lambda$ 's, $\Xi$ 's and $\Omega$ 's are well described in the $\mathrm{p}-\mathrm{Pb}$ case by the present model. Strangeness enhancement is predicted in the present calculation for $\mathrm{Pb}-\mathrm{Pb}$ due to rescattering. However, for central $\mathrm{Pb}-$ $\mathrm{Pb}$ collisions the experimentally observed hyperon yields are underestimated by the present calculations. The discrepancy to the data increases strongly with the strangeness content of the hadron.

An ad hoc overall increase of the color electric field strength (effective string tension of $\kappa=3 \mathrm{GeV} / \mathrm{fm}$ ), or, equivalently, a reduction of the constituent quark masses to the current quark masses enhances the hyperon yields to the experimentally observed high values.

Enhancement factors of $\approx 1.5(2)$ for $\Lambda$ 's, $\approx 2(6)$ for $\Xi^{-}$'s, and $\approx 5(13)$ for $\Omega^{-}$'s are obtained at midrapidity. The values in brackets are the results of the reduced masses/enhanced string tension calculations. The enhancement depends strongly on rapidity.

The reconstructed yields of strange resonances are shown to be strongly affected by the rescattering of their decay products. $\phi$ yields are reduced by $\approx 25 \%$ due to kaon rescattering, $\Lambda^{*}(1520)$ yields are even reduced by $\approx 50 \%$ ! As a consequence the observed differences of the inverse slope parameters $T$ of $\phi$ mesons (NA40 vs. NA50) can be explained qualitatively.

Complementary, also the late resonance production via hadronic rescattering processes plays an important role, e.g. $\mathrm{K}^{+} \mathrm{K}^{-} \rightarrow \phi$.

\section{The UrQMD model - one test}

The Ultrarelativisitc Quantum Molecular Dynamics model (UrQMD) has been developed to simulate heavy ion collisions in a wide energy range (from GSI/SIS to $\mathrm{BNL} / \mathrm{RHIC}$ ). A detailed documentation of the underlying concepts of the model and comparisons to experimental data are available in [9, 10]. The two main constituents of the model are on the one hand the particles that are incorporated and propagated and on the other hand the cross sections for the various collision processes. On the order of one hundred known ground and excited baryon and meson states are in the model in addition to the higher mass states modeled via string degrees of freedom. The string tension $\kappa$ is set to $\kappa=1 \mathrm{GeV} / \mathrm{fm}[11]$. Discussing the strangeness production via string excitation and fragmentation $[11]$ the production probability of a $s \bar{s}$ pair is reduced as compared to $u \bar{u} / d \bar{d}$-pairs according to the Schwinger formula 12]

$$
\gamma_{s}=\frac{P(s \bar{s})}{P(q \bar{q})}=\exp \left(-\frac{\pi\left(m_{s}^{2}-m_{q}^{2}\right)}{2 \kappa}\right) .
$$

Here, the masses of the strange $m_{s}$ and the up/down $m_{q}$ quarks enter. The constituent quark mass values $m_{q}=0.3 \mathrm{GeV}$ and $m_{s}=0.5 \mathrm{GeV}$ yield a strangeness suppression factor $\gamma_{s} \approx 0.3$.

One of several tests of the model is to demonstrate that the particle production yields are reproduced reasonably in elementary collisions. Fig.1 and Fig.2 show the comparisons of $\pi^{ \pm}, \mathrm{K}^{ \pm}, \Lambda$ and $\bar{\Lambda}$ yields to experimental data in proton proton collisions as a function of the center of mass energy. The shown agreement over a large energy 
range supports the chosen set of model parameters, e.g. the parameters for the string decay, and provides a good starting point to investigate nucleus nucleus collisions. The $\bar{\Lambda}$ 's might be slightly overpredicted by the model calculations but in this case the data show uncertainties as well (Fig.2).

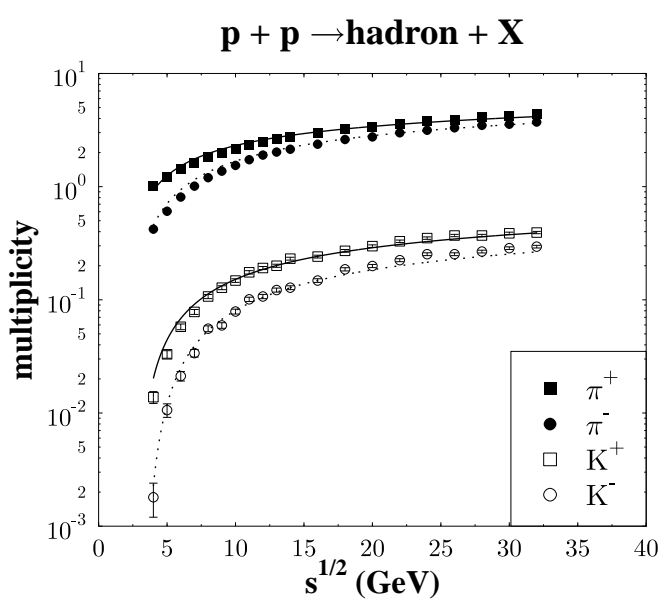

Figure 1: Multiplicities of $\pi^{ \pm}$and $\mathrm{K}^{ \pm}$in pp collisions as a function of the center of mass energy $\sqrt{s}$. The symbols show results of the UrQMD calculations and lines represent parametrizations of experimental data.

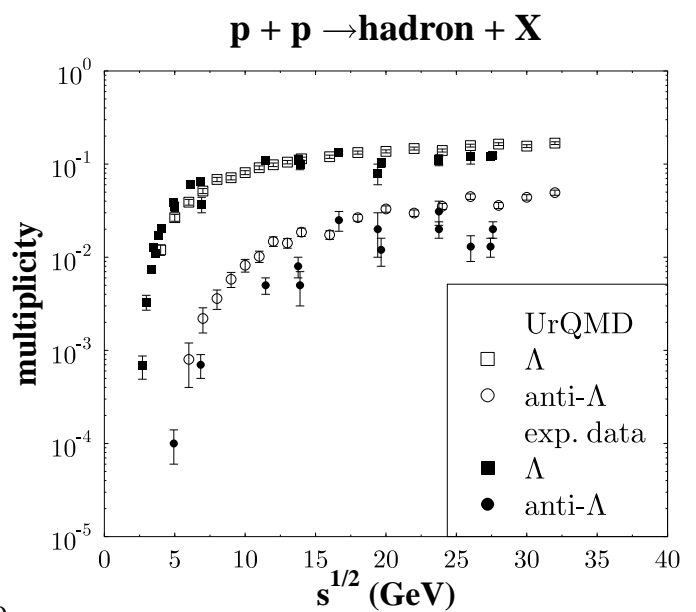

Figure 2: Multiplicities of $\Lambda$ and $\bar{\Lambda}$ in pp collisions as a function of the center of mass energy $\sqrt{s}$. Open symbols show UrQMD results, full symbols are experimental data.

\section{Comparison to data and reduced quark masses}

Fig. 3 shows (anti-)hyperon yields in $\mathrm{Pb}(158 \mathrm{AGeV}) \mathrm{Pb}$ collisions (as a function of the number of participants) and in $\mathrm{pPb}$ collisions [2]. While the agreement between data of WA97 (stars) and UrQMD (open symbols) seems to be reasonable in $\mathrm{pPb}$ collisions discrepancies are observed in central collisions that increase with the strangeness content of the probe. Motivated by chiral symmetry restoration the quark masses may be reduced that enter in (1) thus enhancing the strangeness suppression factor $\gamma_{s}$ [2]. A reduction of the constituent quark masses to the current quark masses $m_{q}=10 \mathrm{MeV}$ and $m_{s}=230 \mathrm{MeV}$ yields $\gamma_{s} \approx 0.65$ [2]. Similarly, the superposition of several strings may increase the color electric field strength [13, 14, thus leading to an effectively enhanced string tension. Enhancing the string tension to $\kappa=3 \mathrm{GeV} / \mathrm{fm}$ yields $\gamma_{s} \approx 0.65$, too [2]. The reduced masses/enhanced string tension UrQMD calculations enhance the calculated yields to the data as shown in Fig.3 (full symbols). Since the asymmetric $\mathrm{p}+\mathrm{Pb}$ collisions are not ideally suited for the comparison to central $\mathrm{Pb}+\mathrm{Pb}$ collisions (e.g. asymmetric rapidity distributions [2]) the enhancement factors $E_{Y}$ of the hyperons $Y$ are determined by $E_{Y}=\left(Y / \pi^{-}\right)_{\text {cent }} /\left(Y / \pi^{-}\right)_{\text {peri }}$ in $\mathrm{Pb}+\mathrm{Pb}$. As shown in Fig.4 they reach their maximum at midrapidity. Open symbols are the enhancement factors for the UrQMD calculations while full symbols show the results if the reduced masses/enahnced string tension calculations are taken into account for the central collisions. For the $\Omega$ 's the enhancement factor can be as large as 15 (only for the reduced masses/enhanced string tension case). A microscopic analysis shows that most of the $\Omega$ 's are produced in secondary meson baryon collisions $(\approx 80 \%)$ (e.g. meson $\Lambda$, meson $\Xi$ ) whereas baryon baryon collisions contribute on the order of $20 \%$ 
(e.g. nucleon nucleon, nucleon $\Lambda$, nucleon $\Xi$ ). About $15 \%$ of the produced $\Omega$ 's are again absorbed (e.g. by $\Omega K, \Omega \bar{B}$ ).

p, Pb (158 AGeV) Pb

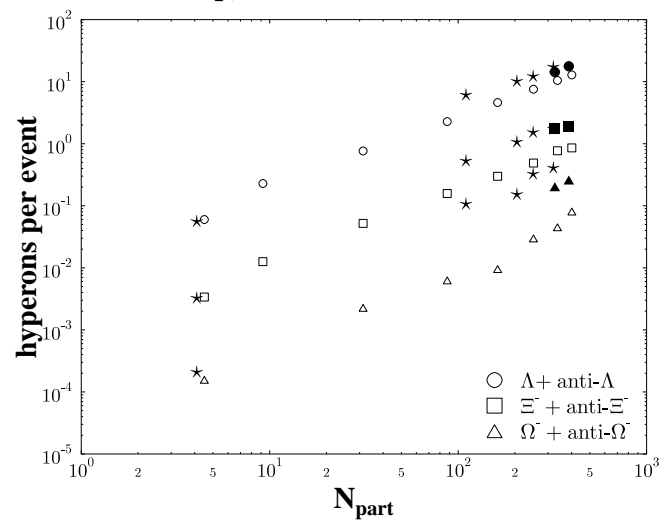

\section{Pb (158 AGeV) Pb}

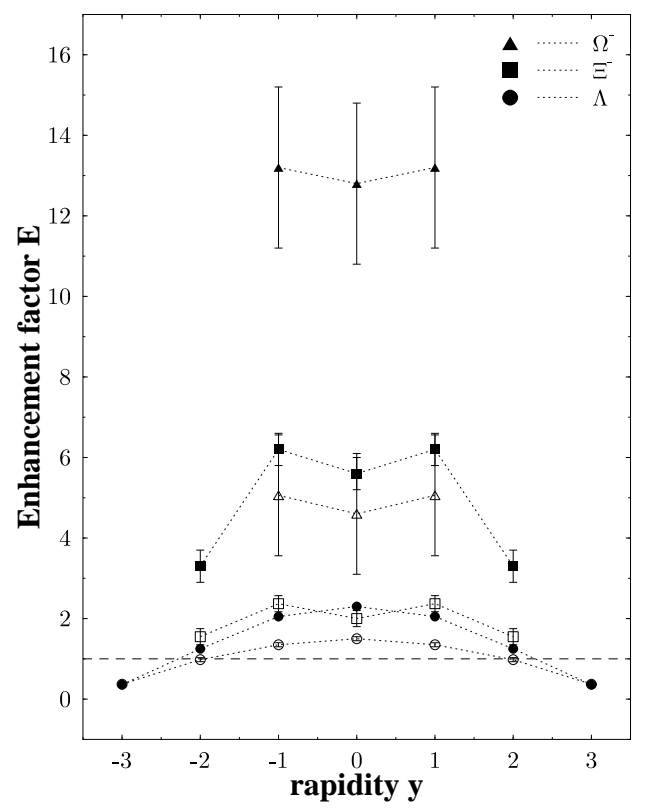

Figure 3: Multiplicities of (anti-)hyperons per event at midrapidity $\left|y-y_{\mathrm{cm}}\right|<0.5$ as a function of the number of participants $N_{\text {part }}$ for $\mathrm{Pb}-\mathrm{Pb}$ and $\mathrm{p}-\mathrm{Pb}$ collisions at $158 \mathrm{AGeV}$. Stars are data of WA97, open symbols are UrQMD results and full symbols are UrQMD results with reduced masses or enhanced string tension.

Figure 4: Enhancement factors $E_{Y}$ for $\Lambda$ 's (circles), $\Xi$ 's (squares) and $\Omega$ 's (triangles) as a function of rapidity in $\mathrm{Pb}(158 \mathrm{AGeV}) \mathrm{Pb}$ collisions. Open symbols show UrQMD calculations, full symbols represent results when the reduced masses/enhanced string tension calculations are taken for the central collisions. $E_{\Omega^{-}}$may reach a value of 15 ! Strangeness suppression is predicted for $\Lambda$ 's at target/projectile rapidity.

In order to support the line of arguments that lead to the reduction of quark masses Fig.5 shows the baryonic particle densities as a function of temperature as obtained from a chiral $S U(3) \times S U(3) \sigma-\omega$ model 115 . The model selfconsistently predicts a phase transition for $T \approx 150 \mathrm{MeV}$ and $\mu=0$ [15]. At this point the masses of the baryons drop drastically and this leads to the strong enhancement of baryonic particle (and antiparticle) densities. 


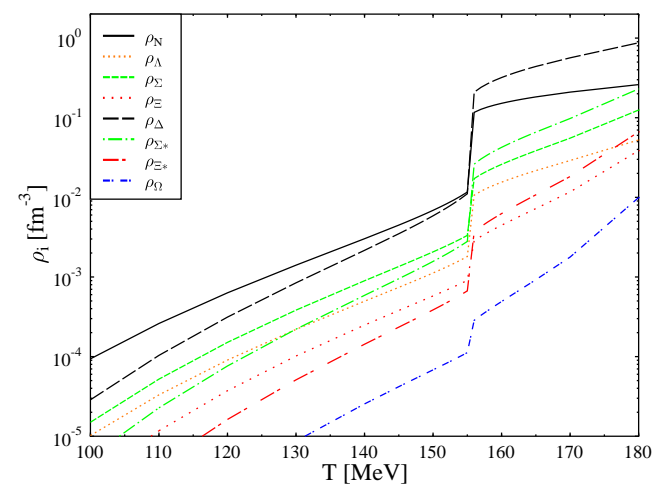

Figure 5: Baryonic particle densities as a function of temperature for vanishing chemical potential in a chiral $\mathrm{SU}(3) \times \mathrm{SU}(3) \quad \sigma-\omega$ model. Due to the phase transition at $T \approx$ $150 \mathrm{MeV}$ and the strong decrease of the baryonic masses, the particle and antiparticle densities strongly increase around the phase transition region.

\section{Strange resonance yields $-\Lambda^{*}(1520)$ and $\phi$}

In this section we briefly discuss the production of the $\Lambda^{*}(1520)$ resonance and the $\phi$ meson and how the reconstruction of these particles by their hadronic decay products is distorted due to rescattering.

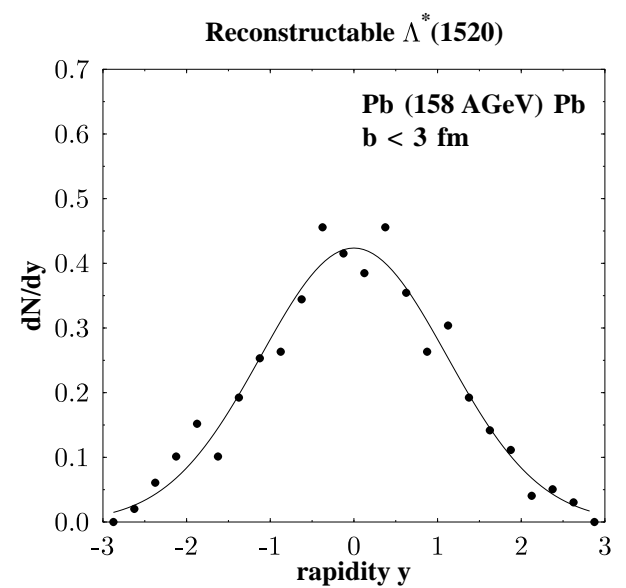

Figure 6: Rapidity distribution $d N / d y$ of reconstructable $\Lambda^{*}(1520)$ 's, $\quad$ i.e. $\quad \Lambda^{*}(1520)$ 's whose decay products do not rescatter, in central $(b<3 \mathrm{fm})$ $\mathrm{Pb}(158 A \mathrm{GeV}) \mathrm{Pb}$ collisions as predicted by the UrQMD model. The total number of $\Lambda^{*}(1520)$ decays is about twice the number of reconstructable $\Lambda^{*}(1520)$ 's.

The $\Lambda^{*}(1520) \quad\left(I\left(J^{P}\right)=0\left(\frac{3}{2}^{-}\right)\right)$is currently investigated by the NA49 collaboration 16] in $\mathrm{Pb}(158 \mathrm{AGeV}) \mathrm{Pb}$ collisions. Its free width is $\Gamma \approx 15.6 \mathrm{MeV}$ corresponding to $\tau \approx 12.6 \mathrm{fm} / \mathrm{c}$. The main decay channels are $N \bar{K}(45 \%), \Sigma \pi(42 \%)$, and $\Lambda \pi \pi(10 \%)$. Especially the $\Lambda^{*}(1520) \rightarrow p K^{-}$decay is suited for the reconstruction by invariant mass distributions. The UrQMD calculations predict $\approx 2.4 \Lambda^{*}(1520)$ decays per central $\mathrm{Pb}+\mathrm{Pb}$ collision (integrated over all decay channels). If this is multiplied with the branching ratio to the $p K^{-}$channel $(22.5 \%)$ and the experimental acceptance $(\approx 50 \%)$ an experimental detection probability of about $0.25 \Lambda^{*}(1520)$ per event is predicted. However, subsequent collisions of the decay products (e.g. $K^{-} p$ ) have not been taken into account for this estimate. They destroy the signal in the invariant mass spectra, thus lowering the observable yield. Fig.5 shows the rapidity distribution of those $\Lambda^{*}(1520)$ whose decay products do not suffer subsequent collisions. Its width is $\sigma_{\mathrm{FWHM}} \approx 2.5$. Integrating this rapidity distribution yields a value of $\approx 1.2 \Lambda^{*}(1520)$ per event. As a consequence, only half of the produced 
$\Lambda^{*}(1520)$ could be ideally reconstructed by their decay products $x$ and $y$

$$
\frac{\Lambda^{*} \rightarrow(x y)_{\text {escape }}}{\Lambda^{*} \rightarrow(x y)_{\text {all }}} \leq 0.5 .
$$

If an enhancement of the $\Lambda^{*}$ 's due to a reduction of the quark masses/enhanced string tension is taken into account the above yields increase by a factor 1.5, corresponding to the enhancement factors of K's and $\Lambda^{\prime}$ 's with the same strangeness content.

Similarly, $\phi$ meson yields and their reconstruction via $\phi \rightarrow K^{+} K^{-}$are affected due to rescattering of the kaons. In this case the ratio of reconstructable to total $\phi$ decays is larger than in the $\Lambda^{*}(1520)$ case (mostly due to the longer lifetime of the $\phi$ )

$$
\frac{\phi \rightarrow(x y)_{\text {escape }}}{\phi \rightarrow(x y)_{\text {all }}} \leq 0.75 \text {. }
$$

However, this approximate $25 \%$ decrease of the observable yield improves the discrepancies between data and microscopic model predictions (e.g. [2]). The rapidity distributions show that the decay products are preferentially rescattered around midrapidity thus broadening the reconstructable $\phi$ rapidity distribution. Complementary the decay products are rescattered preferentially at low transverse momenta thus pretending a larger inverse slope parameter, as also shown in [20]. This corresponds to the different measured inverse slope parameters of the NA49 $\left(\phi \rightarrow K^{+} K^{-}\right)$and NA50 $\left(\phi \rightarrow \mu^{+} \mu^{-}\right)$collaborations [7, 21, 2]. Contrary, Figure 7: Number of $\phi$ mesons at

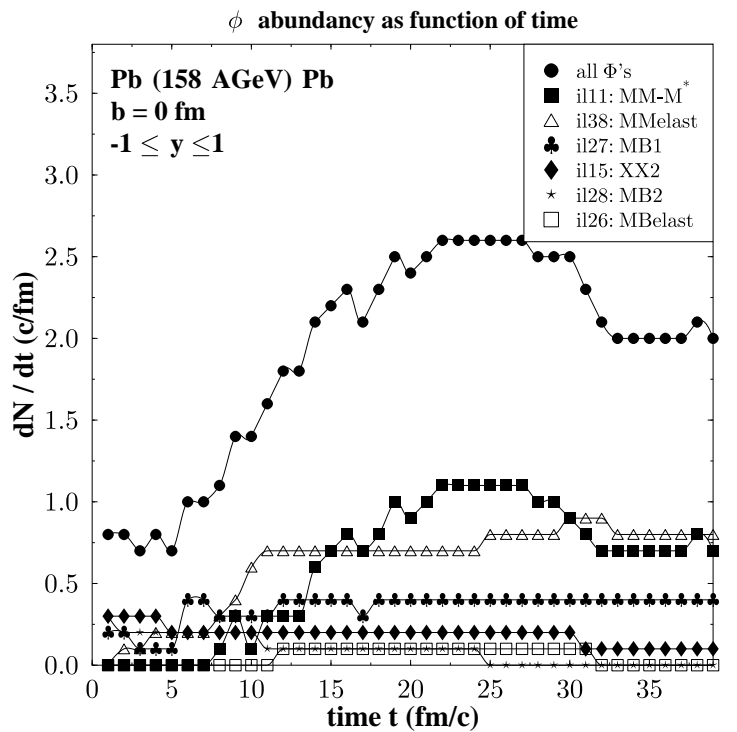
midrapidity as function of time in central $\mathrm{Pb}(158 A \mathrm{GeV}) \mathrm{Pb}$ collisions, calculated with the UrQMD model (circles). The other lines add up to the circles and show the last processes of the $\phi$ 's in each time step. Meson-meson collisions producing a $\phi$ meson (full squares), string excitation and decay producing $\phi$ 's (club,diamond,star) and elastic collisions of $\phi$ 's are depicted. The string excitations and elastic collisions are subdivided into meson-baryon collisions (MB) and meson-meson (MM) collisions leading to the excitation of one (MB1) or two (MB2) strings. (XX2) represents the string excitation by baryon-baryon (mainly first collisions) or meson-meson collisions.

the production of $\phi$ 's is also strongly driven by late kaon kaon scattering as shown in Fig.7. In the first $5 \mathrm{fm} / \mathrm{c} \phi$ production via string decays dominates while in the later stages $(10 \mathrm{fm} / \mathrm{c}<t<20 \mathrm{fm} / \mathrm{c})$ the resonant production via $K \bar{K} \rightarrow \phi$ becomes important. Approximately $40 \%$ of the $\phi$ 's scatter elastically. The strongly timedependent $\phi$ abundancy (due to late resonant meson meson collisions) makes it questionable whether the inclusion of $\phi$ 's in a thermodynamical analysis [17, 18, 19] is reasonable. 


\section{Summary}

- relative enhancement of strange particle production in central $\mathrm{Pb}+\mathrm{Pb}$ collisions with respect to peripheral $\mathrm{Pb}+\mathrm{Pb}$ or $\mathrm{p}+\mathrm{Pb}$ collisions

$\longrightarrow$ enhancement grows with strangeness content of probe

$\longrightarrow$ UrQMD predicts enhancement due to rescattering but not sufficient to explain data

$\longrightarrow$ reduction of quark masses or enhanced string tension reproduces observed high yields

$\longrightarrow$ strong rapidity dependence of enhancement factors

- unstable (strange) particle yields $\left(\Lambda^{*}(1520), \phi\right)$ are distorted due to rescattering of decay products

$\longrightarrow$ approximately $25 \%$ of $\phi$ 's and $50 \%$ of $\Lambda^{*}$ 's have rescattered decay products

- late $\phi$ production by resonant $K \bar{K}$ scattering

\section{Acknowledgments}

This work is supported by DFG, BMBF and the Alexander von Humboldt-Stiftung.

\section{References}

[1] Rafelski J, Müller B Phys. Rev. Lett. 48, (1982) 1066; (E) 56 (1986) 2334; Koch P, Müller B, Rafelski J Phys. Rep. 142, (1986) 167; Koch P, Müller B, Stöcker H, Greiner W Mod. Phys. Lett. A3, (1988) 737

[2] Soff S, Bass S A, Bleicher M, Bravina L, Gorenstein M, Zabrodin E, Stöcker H, Greiner W Phys. Lett. B471, (1999) 89 and refs. therein

[3] Senger P, Ströbele H J. Phys. G: Nucl. Phys. 25, (1999) R59

[4] Strangeness in Quark Matter (Padua, Italy, 1998), J. Phys. G: Nucl. Phys. 25, (1999) 143; Strangeness in Quark Matter (Santorini, Greece, 1997), J. Phys. G: Nucl. Phys. 23, (1997) 1785; Relativistic Aspects of Nuclear Physics (Rio, Brazil, 1995), T. Kodama et al., eds., World Scientific. Strangeness in Hadronic Matter (Budapest, Hungary, 1996), Budapest, Akademiai Kiado. Strangeness in Hadronic Matter (Tucson, AZ, 1995), AIP Conf. 340; Strange Quark Matter in Physics and Astrophysics (Aarhus, Denmark, 1991), Nucl. Phys. B24B, (1991)

[5] Stock R Phys. Lett. B456, (1999) 277

[6] Andersen E et al (WA97 collaboration) Phys. Lett. B433, (1998) 209; Lietava R et al (WA97 collaboration) J. Phys. G: Nucl. Phys. 25, (1999) 181; Caliandro R et al (WA97 collaboration) J. Phys. G: Nucl. Phys. 25, (1999) 171; Margetis S et al (NA49 collaboration) J. Phys. G: Nucl. Phys. 25, (1999) 189

[7] Sikler F et al (NA49 collaboration) Nucl. Phys. A661, (1999) and Höhne C et al (NA49 collaboration) Quark Matter 1999. Nucl. Phys. A661 (1999) 485c

[8] Greiner C, Leupold S nucl-th/0009036

[9] Bass S A, Belkacem M, Bleicher M, Brandstetter M, Bravina L, Ernst C, Gerland L, Hofmann M, Hofmann S, Konopka J, Mao G, Neise L, Soff S, Spieles C, Weber H, Winckelmann L, Stöcker H, Greiner W, Hartnack C, Aichelin J, Amelin N Prog. Part. Nucl. Phys. 41, (1998) 255; Bleicher M, Zabrodin E, Spieles C, Bass S A, Ernst C, Soff S, Bravina L, Belkacem M, Weber H, Stöcker H, Greiner W J. Phys. G: Nucl. Phys. 25, (1999) 1859

[10] Soff S, Dissertation Univ. Frankfurt, Ibidem-Verlag, ISBN 3-89821-074-X, Stuttgart (2000).

[11] Anderson B, Gustafson G, Ingelman G, Sjöstrand T, Phys. Rep. 97, (1983) 31

[12] Schwinger J, Phys. Rev. 82, (1951) 664

[13] Biro T, Nielsen H, Knoll J Nucl. Phys. B245, (1984) 449; Knoll J, Z. Phys. C38, (1988) 187

[14] Sorge H, Berenguer M, Stöcker H, Greiner W, Phys. Lett. B289, (1992) 6

[15] Zschiesche D et al Nucl. Phys. A663, (2000) 737 and refs. therein

[16] Markert $\mathrm{C}$ et al manuscript in preparation and Barton $\mathrm{R}$ et al contribution to this volume

[17] Yen G, Gorenstein M Phys. Rev. C, (1999) 2788 and Gorenstein M, Kostyuk A, Stöcker H, Greiner W hep-ph/0010148

[18] Hamieh S, Redlich K and Tounsi A Phys. Lett. B486 (2000), 61

[19] Rafelski J, Letessier J nucl-th/9903018

[20] Johnson S, Jacak B and Drees A nucl-th/9909075v2

[21] Willis N et al (NA50 collaboration) Quark Matter 1999, Nucl. Phys. A661, (1999) 534c 